ANL-HEP-25

CERN-TH-96-75

ITP-SB-96-17

February 1, 2008

\title{
SUDAKOV FACTORIZATION AND RESUMMATION
}

\author{
Harry Contopanagos ${ }^{1}$, Eric Laenen ${ }^{2}$ and George Sterman $^{3}$ \\ ${ }^{1}$ High Energy Physics Division, Argonne National Laboratory \\ Argonne, IL 60439-4815 \\ ${ }^{2}$ CERN Theory Division \\ CH-1211 Geneva 23, Switzerland \\ ${ }^{3}$ Institute for Theoretical Physics, SUNY Stony Brook \\ Stony Brook, New York 11794-3840
}

\begin{abstract}
We present a unified derivation of the resummation of Sudakov logarithms, directly from the factorization properties of cross sections in which they occur. We rederive in this manner the well-known exponentiation of leading and nonleading logarithmic enhancements near the edge of phase space for cross sections such as deeply inelastic scattering, which are induced by an electroweak hard scattering. For QCD hard-scattering processes, such as heavy-quark production, we show that the resummation of nonleading logarithms requires in general mixing in the space of the color tensors of the hard scattering. The exponentiation of Sudakov logarithms implies that many weighted cross sections obey particular evolution equations in momentum transfer, which streamline the computation of their Sudakov exponents. We illustrate this method with the resummation of soft-gluon enhancements of the inclusive DrellYan cross section, in both DIS and $\overline{\mathrm{MS}}$ factorization schemes.
\end{abstract}

ANL-HEP-25

CERN-TH-96-75

ITP-SB-96-17 


\section{Introduction}

Our aim in this paper is to treat cross sections and amplitudes that allow Sudakov exponentiation and resummation in a unified and streamlined manner. We shall point out the factorization that underlies these resummations, and derive the resummed form directly from it. The close relation of factorization to Sudakov exponentiation was emphasized and exploited long ago by Mueller, ${ }^{[1]}$ Collins and Soper ${ }^{[2]}$ and Sen. ${ }^{[3]}$ Here, we shall generalize this observation, and develop a method that makes the wide range of its application to cross sections and amplitudes straightforward and, we hope, clear.

A classic example ${ }^{[4]}$ of Sudakov exponentiation is the dimensionally regulated electromagnetic form factor of a massless quark ${ }^{[5]}$

$$
\Gamma_{\mu}=\bar{u}\left(p^{\prime}\right) \gamma_{\mu} u(p) F\left(Q^{2}, \epsilon\right)
$$

with $Q^{2}=\left(p^{\prime}-p\right)^{2}$. In QCD, the form factor $F\left(Q^{2}, \epsilon\right)$ exponentiates as $^{[6]}$

$$
F\left(Q^{2}, \epsilon\right)=\exp \left\{-\frac{1}{2} \int_{0}^{Q^{2}} \frac{d \eta^{2}}{\eta^{2}}\left[K\left(\alpha_{s}\left(\mu^{2}\right), \epsilon\right)+G\left(\eta^{2} / \mu^{2}, \alpha_{s}\left(\mu^{2}\right), \epsilon\right)\right]\right\}
$$

where, because $F\left(Q^{2}, \epsilon\right)$ is invariant under renormalization, the functions $K$ and $G$ satisfy

$$
\mu \frac{d K}{d \mu}=-\mu \frac{d G}{d \mu}
$$

Infrared singularities are summarized by $K$, which contains at most a single pole in $\epsilon=2-d / 2$, while $G$, which summarizes ultraviolet behavior, has at most a single logarithm in $\eta^{2} / \mu^{2}$. $K$ and $G$ are each power series in $\alpha_{s}$, which may be read off from low-order calculations. ${ }^{[6]}$ For a fixed coupling, $\ln F\left(Q^{2}\right)$ has double logarithms of $Q^{2},[4]$ and double poles in $\epsilon$, generated from the explicit integrals.

Sudakov exponentiation is much more general than this example, however. It applies as well to many cross sections involving a large momentum transfer, in special limits of their phase spaces. As we shall see, logarithms in these cross sections exponentiate after a Mellin or Fourier transform. We shall refer to this range of possibilities collectively as Sudakov resummation. 
The connection between factorization and resummation is already illustrated by perturbative renormalization, in which the general relation of unrenormalized and renormalized Green functions of fields $\phi_{i}$ carrying momenta $p_{i}$ is

$$
G_{\mathrm{un}}\left(p_{i}, M, g_{0}\right)=\prod_{i} Z_{i}^{1 / 2}(\mu / M, g(\mu)) G_{\mathrm{ren}}\left(p_{i}, \mu, g(\mu)\right)
$$

$M$ is an ultraviolet cutoff, and $g(\mu)$ and $g_{0}$ are the renormalized and bare couplings respectively. The independence of $G_{\text {un }}$ from $\mu$ and $G_{\text {ren }}$ from $M$ may be used to derive renormalization group equations,

$$
\mu \frac{d \ln G_{\mathrm{ren}}}{d \mu}=-\sum_{i} \gamma_{i}(g(\mu))
$$

in which the anomalous dimensions $\gamma_{i}=(1 / 2)(\mu d / d \mu) \ln Z_{i}$ appear as constants in the separation of variables, free of explicit dependence on either $\mu$ or $M$.

Very similar observations apply to structure functions in deeply inelastic scattering (DIS), which we may write in a convolution form, neglecting particle labels, as

$$
F\left(x, Q^{2}\right)=\int_{x}^{1} \frac{d y}{y} C\left(x / y, Q^{2} / \mu^{2}, g(\mu)\right) \phi\left(y, \mu^{2}\right),
$$

where $C$ is a short-distance coefficient function, and $\phi$ is an infrared-sensitive parton distribution. The parameter $\mu$ here plays the role of a "factorization" scale, separating long from short distances. The expression (1.6) factorizes into a product under moments $\int_{0}^{1} d x x^{N-1}$,

$$
\tilde{F}\left(N, Q^{2}\right)=\tilde{C}\left(N, Q^{2} / \mu^{2}, g(\mu)\right) \tilde{\phi}(N, g(\mu))
$$

Since $\tilde{F}$ is physical and thus independent of $\mu$, we again find a simple renormalization group equation for $\tilde{C}$ and $\tilde{\phi}$

$$
\left(\mu \frac{d}{d \mu}-\gamma_{N}(g)\right) \ln \tilde{C}=0=\left(\mu \frac{d}{d \mu}+\gamma_{N}(g)\right) \ln \tilde{\phi}
$$

where the anomalous dimension depends on $N$ and $g$, the only dimensionless parameters shared by the two functions on the right-hand side of (1.7). 
We will show how Sudakov behavior can be derived from a generalization of the singlescale, two-function factorization illustrated by DIS above, to a two-scale, multi-function factorization. ${ }^{[78[9]}$ Such a factorization is characteristic of physical quantities that describe hard scatterings, and which are sensitive to three generic regions of momentum space, associated with three sorts of quanta. These are: off-shell ("hard") partons at short distances, fastmoving on-shell ("collinear") partons near the light cone, and long-wavelength, soft partons. The separation of a cross section into separate functions for each type of excitation requires multiple factorizations. The independence of the final expression from the details of these factorizations, however, leads to a consistency equation, and consequently to Sudakov resummation. This process is related to the use of matching conditions in effective field theories. ${ }^{[10]}$

A number of phenomenologically useful and theoretically important resummations of this type have been derived or postulated for quantities of experimental and theoretical interest, including the Sudakov form factor itself $f^{\left[5111^{3]}\right]}$ the transverse momentum distribution of backto-back particles in $\mathrm{e}^{+} \mathrm{e}^{-}$annihilation ${ }^{[2][1]]}$ and of Drell-Yan pairs, ${ }^{[12]}$ the total Drell-Yan cross section $^{[13][14][15][16][17]}$ and a large class of infrared safe event shapes in $\mathrm{e}^{+} \mathrm{e}^{-}$annihilation, ${ }^{[18]}$ as well as elastic scattering amplitudes at very high energy. ${ }^{[19]}$ Closely related expressions apply to the momentum dependence of vacuum expectations of products of Wilson lines (ordered exponentials $)^{[20]}$. Although all related, the derivations of Sudakov resummations for these quantities have been sufficiently varied in presentation and emphasis that a single derivation from an underlying principle should be useful. In this paper, we shall provide such a derivation based on a common factorization into functions sensitive to hard, collinear and soft partons.

Yet another motivation for studying resummations of this form is that they naturally result in exponentials of integrals over momentum scales of the running coupling. The singularity of the perturbative $\alpha_{s}\left(\mu^{2}\right)$ at $\mu=\Lambda_{\mathrm{QCD}}$ is sometimes referred to as an infrared renormalon ${ }^{[21]}$, whose presence and nature may shed light on power-suppressed corrections to cross sections of interest. Although we shall not investigate renormalons directly in this paper, the generality of Sudakov resummation should help emphasize their relevance.

The factorization into short-distance, jet and soft functions is quite general, applicable to many weighted cross sections with large momentum transfers, ${ }^{[7]}$ and to some elastic amplitudes as well, when considered in impact parameter form. ${ }^{[19]}$ In the following section we shall use this factorization to derive the general Sudakov resummation formula for electroweak- 
induced processes (Drell-Yan, deeply inelastic scattering, $\mathrm{e}^{+} \mathrm{e}^{-}$). This is the central result of this paper, from which we draw applications in Sections 3-5. Section 3 briefly discusses the extension of the formalism to QCD hard scattering ${ }^{[19][22]}$, which shows that exponentiation applies as well to cross sections involving jets and heavy quark production. In Section 4 we discuss the reexpression of our result as an evolution equation in terms of momentum transfer, whose solution is useful for the computation of dimensionally-regularized cross sections and amplitudes. Finally, in Section 5 we use the Drell-Yan and deeply inelastic scattering cross sections as examples.

\section{Resummation from Factorization}

This section begins with a review of the basic properties held in common by all the cross sections (and, with slight modifications, amplitudes) to which Sudakov resummation applies. These properties are summarized in a rather specific but relatively simple factorized expression satisfied by each cross section in a particular limit of its final-state phase space ${ }^{[7]}$. This underlying factorization is illustrated in Fig 1 for $\mathrm{e}^{+} \mathrm{e}^{-}$annihilation (a), the Drell-Yan cross section (b) and DIS (c). The figure is to be thought of as a reduced diagram, showing on-shell lines of the amplitude and its complex conjugate, in cut diagram notation. All lines are massless.

In the figure, electroweak vector bosons of large spacelike or timelike mometum $q, q^{2}=$ $\pm Q^{2}$, are linked to partons through two hard-scattering functions $H$ and $H^{*}$, in the amplitude and the complex conjugate respectively. $H$ and $H^{*}$ depend only on quanta off-shell by order $Q^{2}$. On-shell particles with momenta of order $Q$ fall into two jets, $J_{1}$ and $J_{2}$ of collinear particles. We assume that the final state, represented by the cut of the diagram, approaches an "edge" of phase space, where all finite-energy particles are concentrated within the two jets. We shall also refer to this as an "elastic" limit, although the final states include arbitrary numbers of particles. In this terminology, the diagrams of Fig. 1 represent the most general configurations that contribute at leading power in $Q^{2}$ in the elastic limit. ${ }^{[7]}$

As shown in the figure, jets may be produced at the hard scattering, as in $\mathrm{e}^{+} \mathrm{e}^{-}$annihilation (Fig. 1a), or they may originate with an incoming parton, and hence be identified with a parton distribution, as in the Drell-Yan process (Fig. 1b). In deeply inelastic scattering 
(Fig. 1c), we have one parton distribution and one outgoing jet. In addition, each such cross section involves the emission of soft particles, represented by function $S$. The elastic limit may be isolated quantitatively by introducing an appropriate weight.

By a weight, we refer to a set of functions $w_{n}\left(\left\{k_{i}\right\}\right)$ of the momenta of the particles in each $n$-particle final state, $\left\{k_{i}\right\}, i=1 \ldots n$. The weighted cross section is a sum over all final states, each integrated over its phase space with weight $w_{n}\left(\left\{k_{i}\right\}\right)$. For definiteness, we assume that the $w_{n}$ are dimensionless, and that they vanish in the elastic limit identified above. We also assume the choice of weight is consistent with the cancellation of infrared divergences due to final-state interactions. We shall refer to such a weight as being infrared safe. This requirement is met if the $w_{n}\left(\left\{k_{i}\right\}\right)$ are symmetric in the momenta $k_{i}$ and satisfy the relations ${ }^{[23]}$

$$
w_{n}\left(k_{1}, \ldots(1-\alpha) k_{n-1}, \alpha k_{n-1}\right)=w_{n-1}\left(k_{1}, \ldots k_{n-1}\right)
$$

which ensures that two states that differ by the emission of zero-momentum particles, or by the rearrangement of momenta among collinear particles, have the same weight.

In $n$ th-order perturbation theory, we encounter logarithmic enhancements in the elastic limit on a diagram-by-diagram basis, with $\alpha_{s}^{n}\left[\left(\ln ^{2 n-1} w\right) / w\right]$ typically the most singular behavior at order $\alpha_{s}^{n}$. These are the singularities we shall organize. In dimensionally-regularized perturbation theory, these logarithms appear from the expansion of kinematic combinations such as

$$
\frac{1}{\epsilon} \frac{1}{\left[w Q^{a}\right]^{1+\epsilon}}
$$

with $a=1$ or 2 (and $\epsilon=2-d / 2$ ), examples of which we shall encounter below.

Not every infrared safe weight leads to exponentiation, however ${ }^{[18]}$. We will need to assume in addition that near the elastic limit, the contributions to the weight of particles within the jets and soft function are independent and additive,

$$
w=w_{1}+w_{2}+w_{s}
$$

with corrections that vanish as $w^{2}$ for small $w$. 
Consider the processes of Fig 1. For the $\mathrm{e}^{+} \mathrm{e}^{-}$annihilation cross section, we may identify $w=1-T$, with $T$ the thrust ${ }^{[18]}$, although similar enhancements may occur in other variables as well. For the inclusive Drell-Yan cross section ${ }^{[13]}$, enhancements occur in the variable $w=1-$ $Q^{2} / s \equiv 1-\tau$, while for incusive DIS they occur in $1-x$, with $x$ the Bjorken scaling variable ${ }^{[13]}$. Finally, for the Drell-Yan cross section at measured transverse momentum ${ }^{[12]} d \sigma / d Q^{2} d^{2} Q_{T}$, we may take $w \sim Q_{T} / Q$.

In the elastic limit, we associate with the jets momenta $p_{1}$ and $p_{2}$, with $Q^{2}= \pm\left(p_{1} \pm p_{2}\right)^{2}$ the scale of the hard scattering. For two outgoing jets, as in $\mathrm{e}^{+} \mathrm{e}^{-}$annihilation, we may regard each $p_{i}^{\mu}$ as the lightlike jet momentum in the elastic limit, and $Q^{2}=\left(p_{1}+p_{2}\right)^{2}$. For an incoming jet, as in DIS, $p_{1}$ may be taken as the lightlike momentum of the initial-state parton. In this case, $Q^{2}=-q^{2}=-\left(p_{1}-p_{2}\right)^{2}$.

The general factorization form near $w=0$, applicable to all these cross sections is

$$
\begin{aligned}
\sigma(w)=H\left(p_{1} / \mu, p_{2} / \mu, \zeta_{i}\right) \int & \frac{d w_{1}}{w_{1}} \frac{d w_{2}}{w_{2}} \frac{d w_{s}}{w_{s}} \\
& \times J_{1}\left(p_{1} \cdot \zeta_{1} / \mu, w_{1}(Q / \mu)^{a_{1}}\right) J_{2}\left(p_{2} \cdot \zeta_{2} / \mu, w_{2}(Q / \mu)^{a_{2}}\right) \\
& \times S\left(w_{s}(Q / \mu), v_{i}, \zeta_{i}\right) \delta\left(w-w_{1}-w_{2}-w_{s}\right),
\end{aligned}
$$

with corrections that vanish as powers of $w$. The specific kinematics in (2.4) is defined by

$$
p_{1}=Q^{+} v_{1}, p_{2}=Q^{-} v_{2}, q^{2}= \pm Q^{2}= \pm 2 Q^{+} Q^{-},
$$

with $v_{i}$ dimensionless lightlike vectors. As indicated, we shall take $v_{1}^{\mu}=\delta_{\mu+}, v_{2}^{\mu}=\delta_{\mu-}$ for definiteness. The factorization eq. (2.4) often applies even if the cross section is not infrared safe, but may be factorized according to the standard procedure. ${ }^{[7]}$ In Drell-Yan, for instance, collinear divergences are factored as usual into the jets $J_{i}$, which play the role of parton distributions, as noted above. In (2.4), $J_{i}, S$ and $H$ represent the contributions of the two jets, of the soft quanta not part of the jets, and of the hard quanta, respectively. We have explicitly exhibited overall factors of $1 / w_{i}, 1 / w_{s}$, which we readily derive from power counting for the soft and jet functions in $(2.4){ }^{[13]}$

We may think of the vectors $\zeta_{1}$ and $\zeta_{2}$ as gauge-fixing vectors $\zeta_{i} \cdot A=0$, used to define the jets $J_{i}$ in terms of particular matrix elements. ${ }^{[13]}$ In such axial gauges, the factorization of 
Sudakov double logarithms into the jets is automatic ${ }^{[5]}$. Although the jet definitions may be gauge-dependent, the cross section itself, of course, remains gauge invariant.

The arguments $w_{i}(Q / \mu)^{a_{i}}$ represent the combination of weight and momentum transfer $Q$ through which the jets depend upon the weight. The value of $a_{i}$ in a given cross section depends on the underlying kinematics. In the analysis of the $x \rightarrow 1$ limit of DIS in Ref. [13] (where $w=1-x$ ), for instance, $a=1$ for the initial-state jet and $a=2$ for the final-state jet. We assume (as is the case for all the examples we know of) that the soft function depends only on the variable $w_{s} Q$. In principle, other combinations of $Q$ and $w$-dependence in eq. (2.4) are possible, but we shall not describe them here. Because of their $\zeta_{i}$-dependence, the $J_{i}$ depend on $p_{i} \cdot \zeta_{i}$ and, in general, $\zeta^{2}$. The variables $p_{i} \cdot \zeta_{i}$, which depend on $\zeta_{i}$, are independent of $w_{i} Q^{a_{i}}$, which is defined by kinematics.

The convolution (2.4) factorizes into a simple product under the transformation

$$
\begin{aligned}
\tilde{\sigma}(N)=\int_{0}^{\infty} d w e^{-N w}= & H\left(p_{1} / \mu, p_{2} / \mu, \zeta_{i}\right) \tilde{S}\left(Q / \mu N, v_{i}, \zeta_{i}\right) \sigma(w) \\
& \times \tilde{J}_{1}\left(p_{1} \cdot \zeta_{1} / \mu, Q / \mu N^{1 / a_{1}}\right) \tilde{J}_{2}\left(p_{2} \cdot \zeta_{2} / \mu, Q / \mu N^{1 / a_{2}}\right),
\end{aligned}
$$

where, for instance, $\tilde{J}=\int_{0}^{\infty}(d w / w) \exp [-N w] J$. Singular behavior in the $w \rightarrow 0$ limit is reflected by growth for large values of the conjugate variable $N$. This is the significance of the lower limit on the $w$ integral. For large $N$, contributions from the upper limit are suppressed, and it may be extended to infinity. We consider $N$ as a complex variable, so that (2.6) represents both Laplace and Fourier transforms. For large $|N|$, it serves as well as a representation of the Mellin transform, since for $w \rightarrow 0, \exp [-N w] \sim(1-w)^{N}$, with corrections that vanish as $w$, which is the accuracy of the basic factorization formula (2.4).

In general, the individual functions $H, J_{i}$ and $S$ each require renormalization, which we assume to be multiplicative. Their renormalization scale dependence is determined by a set of anomalous dimensions,

$$
\begin{aligned}
\mu \frac{d}{d \mu} \ln H & =-\gamma_{H}\left(\alpha_{s}\right) \\
\mu \frac{d}{d \mu} \ln J_{i} & =-\gamma_{J_{i}}\left(\alpha_{s}\right) \\
\mu \frac{d}{d \mu} \ln S & =-\gamma_{S}\left(\alpha_{s}\right) .
\end{aligned}
$$


We assume for this discussion that these anomalous dimensions are independent of the moment variable $N$ and of the $\zeta$ 's. The independence of $\tilde{\sigma}(N)$ from the renormalization scale $\mu$ leads to

$$
\gamma_{H}+\gamma_{S}+\sum_{i} \gamma_{J_{i}}=0
$$

that is, the renormalization-dependence of the individual functions must cancel in their product.

In summary, we are concerned here with weighted cross sections that satisfy the short distance-light cone-long distance convolution of eq. (2.4) in terms of functions that are renormalizable according to eq. (2.7). It seems likely that (2.4) has an interpretation in the language of effective field theories, ${ }^{[10]}$ applied to massless particles in Minkowski space.

We emphasize that in this paper we shall not prove eq. (2.4) and its related renormalization conditions (2.7) for any process, nor do we wish to minimize the challenge involved in carrying out such a proof in any particular case. For the status of such proofs, we refer the reader to the literature cited above. We shall simply assume the result, and show that it is restrictive enough to imply very specific resummation formulas for the small-w limit.

In the arguments below, we shall treat the choices of $\zeta_{i}$ as analogous to an ambiguous choice of renormalization scheme. Like a choice of factorization scale, this very ambiguity results in strong restrictions on the cross section.

The general factorization eq. (2.4), then, cannot depend on the precise choice of $\zeta_{1}$ or $\zeta_{2}$. A small change of $\zeta_{1}$, for instance, leads to a relation between the first-order changes in $H$, $\tilde{S}$ and $\tilde{J}_{1}$, which must cancel. Thus, under a variation of $p_{1} \cdot \zeta_{1}$ at fixed $\zeta_{1}^{2}$ (and any other implicit $\zeta_{1}$-dependence) in the functions, we have the relation

$$
0=\left(p_{1} \cdot \zeta_{1} \frac{\partial}{\partial p_{1} \cdot \zeta_{1}} H\right) \tilde{J}_{1} \tilde{J}_{2} \tilde{S}+H\left(p_{1} \cdot \zeta_{1} \frac{\partial}{\partial p_{1} \cdot \zeta_{1}} \tilde{J}_{1}\right) \tilde{J}_{2} \tilde{S}+H \tilde{J}_{1} \tilde{J}_{2}\left(p_{1} \cdot \zeta_{1} \frac{\partial}{\partial p_{1} \cdot \zeta_{1}} \tilde{S}\right)
$$

$\left(\tilde{J}_{2}\right.$ is independent of $\left.\zeta_{1}\right)$ or equivalently,

$$
\begin{array}{r}
p_{1} \cdot \zeta_{1} \frac{\partial}{\partial p_{1} \cdot \zeta_{1}} \ln \tilde{J}_{1}\left(p_{1} \cdot \zeta_{1} / \mu, Q / \mu N^{1 / a_{1}}\right)=-p_{1} \cdot \zeta_{1} \frac{\partial}{\partial p_{1} \cdot \zeta_{1}} \ln H\left(p_{1} / \mu, p_{2} / \mu, \zeta_{i}\right) \\
-p_{1} \cdot \zeta_{1} \frac{\partial}{\partial p_{1} \cdot \zeta_{1}} \ln \tilde{S}\left(Q / \mu N, v_{i}, \zeta_{i}\right)
\end{array}
$$


The logarithmic derivative of $\tilde{J}_{1}$ with respect to $p_{1} \cdot \zeta_{1}$ may depend on the hard scale $Q \sim p_{1} \cdot \zeta_{1}$, or the soft scale $Q / N$. From (2.10), this dependence is additive in terms of one function that depends on the common variables of $\tilde{J}_{1}$ and $H$ and another that depends on the common variables of $\tilde{J}_{1}$ and $\tilde{S}$. For $H$ these are $\alpha_{s}\left(\mu^{2}\right)$ and $p_{1} \cdot \zeta_{1} / \mu$. For $\tilde{S}$ they are $\alpha_{s}\left(\mu^{2}\right)$ and another variable that depends upon $a_{1}$, given by

$$
\frac{1}{\left(v_{i} \cdot \zeta_{i}\right)^{a_{1}-1}} \frac{Q}{\mu N}=\frac{Q^{a_{1}}}{\left(p_{i} \cdot \zeta_{i}\right)^{a_{1}-1} \mu N} \equiv \frac{Q_{a_{1}}^{\prime}}{\mu N}
$$

where the final form serves to define $Q_{a_{1}}^{\prime}$.

We may thus write $(2.10)$ as

$$
p_{1} \cdot \zeta_{1} \frac{\partial}{\partial p_{1} \cdot \zeta_{1}} \ln J_{1}\left(p_{1} \cdot \zeta_{1} / \mu, Q / \mu N^{1 / a_{1}}\right)=G\left(p_{1} \cdot \zeta_{1} / \mu, \alpha_{s}\left(\mu^{2}\right)\right)+K\left(Q_{a_{1}}^{\prime} / \mu N, \alpha_{s}\left(\mu^{2}\right)\right)
$$

in which the function $G$ matches the variation of the hard part and $K$ the variation of the soft part,

$$
\begin{aligned}
& G=-p_{1} \cdot \zeta_{1} \frac{\partial}{\partial p_{1} \cdot \zeta_{1}} \ln H \\
& K=-p_{1} \cdot \zeta_{1} \frac{\partial}{\partial p_{1} \cdot \zeta_{1}} \ln \tilde{S}
\end{aligned}
$$

Eq. (2.12) is the basic consistency condition referred to in the introduction.

Because $J$ is multiplicatively renormalized, the combination $G+K$ is itself a renormalization invariant $^{[2][3]}$

$$
p_{1} \cdot \zeta_{1} \frac{d}{d p_{1} \cdot \zeta_{1}} \gamma_{J_{1}}\left(\alpha_{s}\right)=\mu \frac{d}{d \mu}\left[G\left(p_{1} \cdot \zeta_{1} / \mu, \alpha_{s}\left(\mu^{2}\right)\right)+K\left(Q_{a_{1}}^{\prime} / \mu N, \alpha_{s}\left(\mu^{2}\right)\right)\right]=0
$$

or, again separating variables, ${ }^{[2]}$

$$
\begin{aligned}
& \mu \frac{d}{d \mu} K\left(Q_{a_{1}}^{\prime} / N \mu, \alpha_{s}\left(\mu^{2}\right)\right)=-\gamma_{K}\left(\alpha_{s}\left(\mu^{2}\right)\right), \\
& \mu \frac{d}{d \mu} G\left(p_{1} \cdot \zeta_{1} / \mu, \alpha_{s}\left(\mu^{2}\right)\right)=\gamma_{K}\left(\alpha_{s}\left(\mu^{2}\right)\right),
\end{aligned}
$$

with $\gamma_{K}$ a Sudakov anomalous dimension. This relation allows us to reexpress $G+K$ in a 
form which relates the scales $p_{1} \cdot \zeta_{1}$ and $Q_{a_{1}}^{\prime} / N$,

$$
\left.K\left(Q_{a_{1}}^{\prime} / N \mu, \alpha_{s}\left(\mu^{2}\right)\right)\right)+G\left(p_{1} \cdot \zeta_{1} / \mu, \alpha_{s}\left(\mu^{2}\right)\right)=-\int_{Q_{a_{1}}^{\prime} / N}^{p_{1} \cdot \zeta_{1}} \frac{d \mu^{\prime}}{\mu^{\prime}} A\left(\alpha_{s}\left(\mu^{\prime 2}\right)\right)+A^{\prime}\left(\alpha_{s}\left(\left(p_{1} \cdot \zeta_{1}\right)^{2}\right)\right)
$$

where the functions $A$ and $A^{\prime}$ are defined by

$$
A\left(\alpha_{s}\right)=\gamma_{K}\left(\alpha_{s}\right)+\beta(g) \frac{\partial}{\partial g} K\left(1, \alpha_{s}\right)
$$

and

$$
A^{\prime}\left(\alpha_{s}\right)=K\left(1, \alpha_{s}\right)+G\left(1, \alpha_{s}\right)
$$

A solution to both (2.12) and the renormalization group equation for the jets in (2.7), which organizes both $p_{i} \cdot \zeta_{i^{-}}$and $N$-dependence is

$$
\begin{aligned}
\tilde{J}\left(p \cdot \zeta / \mu, Q / \mu N^{1 / a},\right. & \left.\alpha_{s}\left(\mu^{2}\right)\right)=\tilde{J}\left(1,1, \alpha_{s}\left(Q^{2} / N^{2 / a}\right)\right) \exp \left[-\int_{Q / N^{1 / a}}^{\mu} \frac{d \lambda}{\lambda} \gamma_{J}\left(\alpha_{s}\left(\lambda^{2}\right)\right)\right] \\
& \times \exp \left[-\int_{Q / N^{1 / a}}^{p \cdot \zeta} \frac{d \lambda}{\lambda}\left\{\int_{Q^{a} / \lambda^{a-1} N}^{\lambda} \frac{d \xi}{\xi} A\left(\alpha_{s}\left(\xi^{2}\right)\right)-A^{\prime}\left(\alpha_{s}\left(\lambda^{2}\right)\right\}\right],\right.
\end{aligned}
$$

where we have used the definition in (2.11) for $Q_{a}^{\prime}$.

In the following, we take $p \cdot \zeta=Q$. Shifts of this value for the form $p \cdot \zeta=C Q$, with $C$ a constant, may be absorbed into redefinitions of $A^{\prime[2]}$. Finally, using the renormalization group equations (2.7) for $H$ and $S$, and the relation (2.8) between their anomalous dimensions, we 
find

$$
\begin{aligned}
\ln \tilde{\sigma}(N)=D_{1}\left(\alpha_{s}\left(Q^{2}\right)\right) & +D_{2}\left(\alpha_{s}\left(Q^{2} / N^{2}\right), a\right) \\
& -2 \int_{Q / N^{1 / a}}^{Q} \frac{d \lambda}{\lambda}\left[\int_{Q^{a} / \lambda^{a-1} N}^{\lambda} \frac{d \xi}{\xi} A\left(\alpha_{s}\left(\xi^{2}\right)\right)-B\left(\alpha_{s}\left(\lambda^{2}\right)\right)\right] \\
= & D_{1}\left(\alpha_{s}\left(Q^{2}\right)\right)+D_{2}\left(\alpha_{s}\left(Q^{2} / N^{2}\right), a\right) \\
& -2 \int_{Q / N^{1 / a}}^{Q} \frac{d \xi}{\xi}\left[\ln (Q / \xi) A\left(\alpha_{s}\left(\xi^{2}\right)\right)-B\left(\alpha_{s}\left(\xi^{2}\right)\right)\right] \\
& -\frac{2}{a-1} \int_{Q / N}^{Q / N^{1 / a}} \frac{d \xi}{\xi} \ln (\xi N / Q) A\left(\alpha_{s}\left(\xi^{2}\right)\right)
\end{aligned}
$$

where for simplicity we have taken $a_{1}=a_{2}=a$, and where

$$
\begin{aligned}
D_{1}\left(\alpha_{s}\left(Q^{2}\right)\right) & =\ln H\left(1,1, \alpha_{s}\left(Q^{2}\right)\right), \\
D_{2}\left(\alpha_{s}\left(Q^{2} / N^{2}\right), a\right) & =\ln \tilde{S}\left(1, \alpha_{s}\left(Q^{2} / N^{2}\right)\right)+\sum_{i=1,2} \ln \tilde{J}_{i}\left(1,1, \alpha_{s}\left(Q^{2} / N^{2 / a}\right)\right) \\
& -\int_{Q / N}^{Q /(N)^{1 / a}} \frac{d \lambda}{\lambda} \gamma_{S}\left(\alpha_{s}\left(\lambda^{2}\right)\right), \\
B\left(\alpha_{s}\right) & =\frac{1}{2} \gamma_{H}+A^{\prime} .
\end{aligned}
$$

Note that the last term on the right-hand side of (2.20) vanishes for $a=1$, in spite of its overall factor of $1 /(a-1)$. Also, we note that in cross sections involving partons in the initial state, such as DIS and Drell-Yan, the function $D_{2}$ will in general contain collinear singularities $(1 / \epsilon$ in dimensional regularization).

At one loop in $\alpha_{s}$, denoting $f\left(\alpha_{s}\right)=\left(\alpha_{s} / \pi\right) f^{(1)}+\ldots$, we have

$$
\ln \tilde{\sigma}(N)=D_{1}^{(0)}+D_{2}^{(0)}+\left(\alpha_{s} / \pi\right)\left[D_{1}^{(1)}+D_{2}^{(1)}-A^{(1)}\left(\frac{1}{a}\right) \ln ^{2} N+2 B^{(1)}\left(\frac{1}{a}\right) \ln N\right] .
$$

Thus, from the one-loop cross section the functions $A$ and $B$ and the combination $D_{1}+D_{2}$ may be simply read off. Higher orders in the functions are similarly determined to higher loop order. 
The result (2.20) shows that the cross section is an exponential, whose exponent has at most double logarithms of the weight variable $N$ for fixed coupling, with higher powers of $N$ generated only by expansion of the running coupling. This is the basic Sudakov exponentiation, in the form derived, for example, by Collins and Soper ${ }^{[2]}$, and in Ref. [19]. The $N$-dependence of couplings in the function $D_{2}$ is, of course, consistent with these results. These formulas, however, must be restricted to values of $N$ for which $\alpha_{s}\left(Q^{2} / N^{2}\right)$ remains well-defined. To go beyond this region, at least formally, we shall use the solution to an evolution equation in momentum transfer, which we shall derive in Section 4 below. Before doing so, however, we observe how these results may be generalized to purely QCD hard-scattering processes, such as inclusive heavy-quark or jet production.

\section{Sudakov Resummation for QCD Hard Scattering}

The methods of Section 2 above apply rather directly to processes that proceed through color exchange, such as heavy quark production ${ }^{[24]}{ }^{[22]}$. To our knowledge, however, until recently $^{[22]}$ the only treatments beyond leading logarithm in the literature for QCD hardscattering processes were for the independent-scattering description of hadron-hadron elastic scattering, ${ }^{[19]}$ and for the scattering of Wilson lines. ${ }^{[25]}$

In place of eq. (2.4), we now have a somewhat more general form, in which there may be more than two jets, and in which the hard and soft scattering functions are labelled by their color content,

$$
\begin{aligned}
\tilde{\sigma}(N) & =\int_{0}^{\infty} d w e^{-N w} \sigma(w) \\
& =\sum_{I J} H_{I J}\left(p_{1} / \mu, p_{2} / \mu, \zeta_{i}\right) \tilde{S}_{I J}\left(Q / \mu N, v_{i}, \zeta_{i}\right) \prod_{i} J_{i}\left(p_{i} \cdot \zeta_{i} / \mu, Q / \mu N^{1 / a_{i}}\right) .
\end{aligned}
$$

The indices $I J$ refer to the color structure of the hard scattering. Because soft gluons decouple from the jet functions, the jets are diagonal in color after factorization, just as for parton distributions. There are two indices in the typical cross section that describes a single hard scattering, one from the amplitude and one from its complex conjugate. For the high- $Q^{2}$ elastic scattering amplitudes of protons, there are as many as three color indices, one for 
each of three quark-quark scattering amplitudes. When the scattering involves quarks, these color factors may be described by singlet or octet exchange.$^{[19[25]}$ Quark-gluon and gluon-gluon scattering may be labelled correspondingly.

For heavy quark production, ${ }^{[22]}$ when the heavy quark mass is of the order of $x_{1} x_{2} s$, with $x_{i}$ partonic momentum fractions, there are only two jet factors, one for each of the incoming partons. The heavy quark propagators involve no collinear divergences (and hence no Sudakov logarithms). They may thus be absorbed, in eikonal approximation, into the soft tensor $S_{I J}$. The dynamics of the heavy quarks beyond eikonal approximation may be absorbed into $H_{I J}$. For two-jet production near the elastic limit, there are four $J_{i}$, two representing incoming partons, and two representing the outgoing jets.

The analysis of Section 2 may be repeated for (3.1), leading to an analog of the resummed result (2.20), except that now, because the anomalous dimensions corresponding to the hard and soft functions are color matrices, we find ordered exponentials for nonleading logarithms. To be specific, the analogs of eqs. (2.7) for anomalous dimensions are

$$
\begin{aligned}
\mu \frac{d}{d \mu}(\ln H)_{I J} & =\left(\Gamma_{H}\left(\alpha_{s}\right)\right)_{I J} \\
\mu \frac{d}{d \mu} \ln \tilde{J}_{i} & =\gamma_{J_{i}}\left(\alpha_{s}\right), \\
\mu \frac{d}{d \mu} \ln S_{I J} & =\left(\Gamma_{S}\left(\alpha_{s}\right)\right)_{I J} .
\end{aligned}
$$

The independence of $d \sigma / d w$ from the renormalization scale $\mu$ then leads to

$$
\left(\Gamma_{H}\left(\alpha_{s}\right)\right)_{K L}+\left(\Gamma_{S}\left(\alpha_{s}\right)\right)_{K L}+\sum_{i} \gamma_{J_{i}} \delta_{K L}=0
$$

We shall not work out any example ${ }^{[2]}$ of this formalism further here. Clearly, however, up to the matrix structure of the anomalous dimensions, the arguments of the previous section may be repeated. In a basis in which the color anomalous dimensions are diagonal, ${ }^{[19[25]}$ there is a separate exponentiation for each diagonal color structure, to leading logarithm in $N$ for $S_{I J}$, which is next-to-leading logarithm overall. 


\section{Evolution in the Momentum Transfer}

The Sudakov exponentiation in eq. (2.20) leads in turn to an alternate form of resummation, from its dependence on the momentum transfer $Q^{2}$. This approach is particularly natural for electroweak processes, in which the hard scattering is a color singlet.

We begin by taking the derivative of eq. (2.20) with respect to $\ln Q$ to derive the Sudakov evolution equation

$$
\begin{gathered}
Q \frac{d}{d Q} \tilde{\sigma}\left(N, Q^{2}\right)=E_{1}\left(\alpha_{s}\left(Q^{2}\right)\right)+E_{2}\left(\alpha_{s}\left(Q^{2} / N^{2}\right), a\right)-2 \int_{Q / N^{1 / a}}^{Q} \frac{d \xi}{\xi} A\left(\alpha_{s}\left(\xi^{2}\right)\right) \\
+\frac{2}{1-a} \int_{Q / N^{2 / a}}^{Q / N^{1 / a}} \frac{d \xi}{\xi} A\left(\alpha_{s}\left(\xi^{2}\right)\right),
\end{gathered}
$$

where, (suppressing arguments of the functions),

$$
E_{1}=\beta \frac{\partial}{\partial g} D_{1}+2 B, \quad E_{2}=\beta \frac{\partial}{\partial g} D_{2}-2 B .
$$

The structure of this equation is enough to imply an alternate resummed expression, of the sort derived, for instance, in Ref. [13], in which the exponent is expressed as an inverse moment.

Eq. (4.1) shows that to leading power in $N$, any factorizing cross section obeys an evolution equation of the form

$$
Q^{2} \frac{d}{d Q^{2}} \tilde{\sigma}\left(N, Q^{2}\right)=\tilde{W}\left(N, Q^{2}\right) \tilde{\sigma}\left(N, Q^{2}\right)
$$

To be specific we may take $\sigma\left(z, Q^{2}\right)$ to represent, for instance, the Drell-Yan (DY) cross section $\sigma_{\mathrm{DY}}\left(z, Q^{2}\right)$ or a deeply inelastic scattering (DIS) structure function $\mathrm{F}_{\mathrm{DIS}}\left(z, Q^{2}\right)$.

The inverse transform of (4.3) is a convolution, whose precise nature depends on which transform is appropriate to the cross section. To be specific, and to relate to the examples we discuss in the following section, we choose the Mellin transform, which leads to the familiar 
evolution equation $^{[26]}$,

$$
\begin{aligned}
Q^{2} \frac{d}{d Q^{2}} \sigma\left(z, Q^{2}\right) & =\int_{z}^{1} \frac{d z^{\prime}}{z^{\prime}} W\left(z^{\prime}, Q^{2}\right) \sigma\left(z / z^{\prime}, Q^{2}\right) \\
W\left(z, Q^{2}\right) & =\int_{-i \infty}^{i \infty} \frac{d N}{2 \pi i} N^{-z} \tilde{W}\left(N, Q^{2}\right)
\end{aligned}
$$

where in the second line we have shown the formal inverse relation between $W$ and $\tilde{W}$.

Working in $d>4$ dimensions, we can take these quantities to be normalized such that

$$
\tilde{\sigma}\left(z, Q^{2}=0\right)=\delta(1-z)
$$

The physical content of this condition is that for zero momentum transfer there is no radiation when the scattering is through an electroweak current. In more than four dimensions, these conditions are explicitly realized order-by-order in perturbation theory. ${ }^{[6]}$

The solution to eq. (4.3) with boundary condition $\tilde{\sigma}(N, 0)=1$ is simply

$$
\begin{aligned}
\tilde{\sigma}\left(N, Q^{2}\right) & \equiv \tilde{\sigma}\left(N, Q^{2} / \mu^{2}, \alpha\left(\mu^{2}\right), \epsilon\right) \\
& =\exp \left[\int_{0}^{Q^{2}} \frac{d \xi^{2}}{\xi^{2}} \tilde{W}\left(N, \frac{\xi^{2}}{\mu^{2}}, \alpha\left(\mu^{2}\right), \epsilon\right)\right],
\end{aligned}
$$

where as above $d=4-2 \epsilon>4$, and $\alpha$ is defined by

$$
\alpha\left(\mu^{2}\right) \equiv \frac{\alpha_{s}\left(\mu^{2}\right)}{\pi}
$$

In eq. (4.6), we have made explicit the perturbative dependence of $\tilde{W}$ on the renormalization scale $\mu$.

We can now employ the invariance of the physical quantity $W$ under changes in the factorization scale $\mu$. There is considerable freedom in how to proceed. Since the LHS of eq. (4.6) is a renormalization group invariant, so is the exponent, and the function $W\left(z, \xi^{2} / \mu^{2}, \alpha\left(\mu^{2}\right), \epsilon\right)$ 
as well. From eq. (2.20), we easily conclude that the coefficient of $\alpha\left(\mu^{2}\right)^{M}$ in $\tilde{W}(N)$ has powers of $\ln N$ up to $\ln ^{M+1} N$. We can now show that the general form of the coefficient of $\alpha_{s}^{M}\left(\mu^{2}\right)$ in the exponent is

$$
\begin{gathered}
W^{(M)}\left(z, Q^{2} / \mu^{2}, \epsilon\right)=\sum_{j=0}^{M} c_{j}^{(M)}\left(Q^{2} / \mu^{2}, \epsilon\right) \mathcal{D}_{j}(z)+f^{(M)}\left(Q^{2} / \mu^{2}, \epsilon\right) \delta(1-z) \\
+h^{(M)}\left(z, Q^{2} / \mu^{2}, \epsilon\right),
\end{gathered}
$$

where $h^{(M)}(z)$ is regular at $z=1$, and where the plus distributions $\mathcal{D}_{j}(z) \equiv\left[\ln ^{j}(1-z) / 1-z\right]_{+}$ are defined as usual by

$$
\int_{x}^{1} d z g(z)[f(z)]_{+}=\int_{x}^{1} d z(g(z)-g(1)) f(z)-g(1) \int_{0}^{x} d z f(z)
$$

with $g(z)$ an arbitrary but smooth function. To show (4.8), we recall that for large $N$,

$$
\int_{0}^{1} d z z^{N-1} \mathcal{D}_{j}(z)=\sum_{k=0}^{j+1} t_{j k} \ln ^{k} N
$$

with corrections that fall off as $1 / N$, where the matrix $[t]$ is invertible (because it is triangular; see for example Table 1 in [14] for explicit coefficients). Then the plus distributions, $W_{\mathcal{D}}^{(M)}$ in eq. (4.8) have moments

$$
\begin{aligned}
\tilde{W}_{\mathcal{D}}^{(M)}\left(N, Q^{2} / \mu^{2}, \alpha\left(\mu^{2}\right), \epsilon\right) & =\sum_{k=1}^{M+1} \ln ^{k} N \sum_{j=k-1}^{M} c_{j}^{(M)} t_{j k}+\sum_{j=0}^{M} c_{j}^{(0)} t_{j 0} \\
& \equiv \sum_{k=0}^{M+1} b_{k}^{(M)} \ln ^{k} N
\end{aligned}
$$

where $b_{k}^{(M)}=\sum c_{j}^{(M)} t_{j k}$ is a function of $Q / \mu$ and $\epsilon$ in general.

The functions $f^{(M)}$ and $h^{(M)}$ in eq. (4.8) are Mellin transforms of constants and terms behaving as powers of $1 / N$ respectively. Since the renormalization group does not affect the $N$ dependence, the $b_{k}$ 's are RG invariant, and by invertibility of the matrix $[t]$, so are the $c_{j}$ 's. Then the series $f(z)=\sum_{M} f^{(M)}$ and $h(z)=\sum_{M} h^{(M)}$ are also RG invariant. Later we will use this freedom in treating parts of the resummed partonic quantities differently under the renormalization group. 
For now we apply renormalization scale invariance to the full $W$ in eq. (4.6):

$$
W\left(z, \xi^{2} / \mu^{2}, \alpha\left(\mu^{2}\right), \epsilon\right)=W\left(z, 1 / \nu, \alpha\left(\nu \xi^{2} / \mu^{2}, \alpha\left(\mu^{2}\right), \epsilon\right), \epsilon\right)
$$

where we are free to choose $\nu$ to be a function of $z$ (and we shall below). To include all corrections of the form $(\alpha \ln N)^{n}$ in the exponent, we need only up to the two-loop running coupling $^{[14][27]}$. Here we need the dimensionally continued version of $\alpha_{s}$, which we have denoted $\alpha\left(\lambda, \alpha\left(\mu^{2}\right), \epsilon\right)$.

The defining equation of the $d$-dimensional running $\alpha$ is

$$
\lambda^{1-\epsilon} \frac{\partial\left[\lambda^{\epsilon} \alpha\left(\lambda, \alpha\left(\mu^{2}\right), \epsilon\right)\right]}{\partial \lambda}=-b_{2} \alpha^{2}\left(\lambda, \alpha\left(\mu^{2}\right), \epsilon\right)-b_{3} \alpha^{3}\left(\lambda, \alpha\left(\mu^{2}\right), \epsilon\right)
$$

with boundary condition $\alpha\left(\lambda=1, \alpha\left(\mu^{2}\right), \epsilon\right)=\alpha\left(\mu^{2}\right)$. Here $b_{2}=\left(11 C_{A}-2 n_{f}\right) / 12$ and $b_{3}=34 C_{A}^{2} / 48-\left(20 C_{A} / 3+4 C_{F}\right) n_{f} / 32$. The solution, linearized in $b_{3}$, is:

$$
\lambda^{\epsilon} \alpha\left(\lambda, \alpha\left(\mu^{2}\right), \epsilon\right)=\frac{\alpha\left(\mu^{2}\right)}{1-\gamma\left(\lambda^{\epsilon}, \epsilon\right) \alpha\left(\mu^{2}\right)}+\frac{b_{3}}{b_{2}} \frac{\alpha^{2}\left(\mu^{2}\right)}{\left(1-\gamma\left(\lambda^{\epsilon}, \epsilon\right) \alpha\left(\mu^{2}\right)\right)^{2}} f\left(\lambda^{\epsilon}, \alpha\left(\mu^{2}\right), \epsilon\right)
$$

with $\gamma\left(\lambda^{\epsilon}, \epsilon\right) \equiv \frac{b_{2}}{\epsilon}\left(\lambda^{-\epsilon}-1\right), f\left(\lambda^{\epsilon}, \alpha, \epsilon\right)=1-\lambda^{-\epsilon}-\left(1+\frac{\epsilon}{b_{2} \alpha}\right) \ln \left(1-\gamma\left(\lambda^{\epsilon}, \epsilon\right) \alpha\right)$.

Given eq. (4.12), the exponent in eq. (4.6) can now be expanded as a power series in $\alpha\left(\lambda, \alpha\left(\mu^{2}\right), \epsilon\right)$, with $\lambda=\nu \xi^{2} / \mu^{2}$,

$$
\begin{array}{r}
\tilde{\sigma}\left(N, Q^{2} / \mu^{2}, \alpha\left(\mu^{2}\right), \epsilon\right)=\exp \left[\int _ { 0 } ^ { 1 } d z z ^ { N - 1 } \int _ { 0 } ^ { \nu Q ^ { 2 } / \mu ^ { 2 } } \frac { d \lambda } { \lambda } \left\{\alpha\left(\lambda, \alpha\left(\mu^{2}\right), \epsilon\right) W^{(1)}(z, 1 / \nu, \epsilon)\right.\right. \\
\left.\left.+\alpha^{2}\left(\lambda, \alpha\left(\mu^{2}\right), \epsilon\right) W^{(2)}(z, 1 / \nu, \epsilon)\right\}\right] .
\end{array}
$$

In this resummed expression, the exponent is itself in the form of a moment. ${ }^{[13]} \mathrm{A}$ similar analysis may be given for Fourier transforms. ${ }^{[28[29]}$ The $d$-dimensional running coupling $\alpha$ in eq. (4.15) has a usual perturbative ("renormalon" ${ }^{[21]}$ ) singularity at $\lambda=\exp \left[-1 / \alpha b_{2}\right]$, as seen from eq. (4.14). Eq. (4.15) must therefore be considered as a resummation order-by-order in a perturbative expansion of its exponent, without further information on how to treat the running coupling at very low scales. 
The functions $W^{(1)}, W^{(2)}$ can be determined by choosing $\nu=1$ in eq. (4.15), and expanding it to two loops in $\alpha\left(\mu^{2}\right)$,

$$
\begin{aligned}
Q^{2} \frac{\partial}{\partial Q^{2}} \ln \tilde{\sigma}\left(N, Q^{2} / \mu^{2}, \alpha\left(\mu^{2}\right), \epsilon\right)=\alpha\left(Q^{2} / \mu^{2}, \alpha\left(\mu^{2}\right), \epsilon\right) \tilde{W}^{(1)}(N, 1, \epsilon) & \\
& +\alpha^{2}\left(Q^{2} / \mu^{2}, \alpha\left(\mu^{2}\right), \epsilon\right) \tilde{W}^{(2)}(N, 1, \epsilon)
\end{aligned}
$$

in terms of $\alpha_{s}\left(\mu^{2}\right)$, using (from (4.14))

$$
\lambda^{\epsilon} \alpha\left(\lambda, \alpha\left(\mu^{2}\right), \epsilon\right)=\alpha\left(\mu^{2}\right)+\gamma\left(\lambda^{\epsilon}, \epsilon\right) \alpha^{2}\left(\mu^{2}\right) .
$$

We then find for the perturbative expansion of the moments

$$
\tilde{W}^{(1)}(N, 1, \epsilon)=\left(Q^{2} / \mu^{2}\right)^{\epsilon} Q^{2} \frac{\partial}{\partial Q^{2}} \tilde{\sigma}^{(1)}\left(N, Q^{2} / \mu^{2}, \epsilon\right)
$$

and

$$
\begin{gathered}
\tilde{W}^{(2)}(N, 1, \epsilon)=\left(Q^{2} / \mu^{2}\right)^{2 \epsilon}\left\{\frac{\partial}{\partial \ln Q^{2}}\left(\tilde{\sigma}^{(2)}\left(N, Q^{2} / \mu^{2}, \epsilon\right)-\frac{1}{2}\left[\tilde{\sigma}^{(1)}\left(N, Q^{2} / \mu^{2}, \epsilon\right)\right] 2\right)\right. \\
\left.-\gamma\left(\left(Q^{2} / \mu^{2}\right)^{\epsilon}, \epsilon\right) \frac{\partial}{\partial \ln Q^{2}} \tilde{\sigma}^{(1)}\left(N, Q^{2} / \mu^{2}, \epsilon\right)\right\} .
\end{gathered}
$$

These results may be inverted to derive $W^{(1,2)}(z, 1, \epsilon)$. The full functions $W^{(1,2)}\left(z, \xi^{2} / \mu^{2}, \epsilon\right)$ may then be constructed by reexpanding $\alpha\left(\xi^{2} / \mu^{2}, \alpha\left(\mu^{2}\right), \epsilon\right)$ in $\alpha\left(\mu^{2}\right)$ in eq. (4.12), using (4.14) with $\lambda=\xi^{2} / \mu^{2}$,

$$
\begin{aligned}
& W^{(1)}\left(z, \xi^{2} / \mu^{2}, \epsilon\right)=\left(\frac{\mu^{2}}{\xi^{2}}\right)^{\epsilon} W^{(1)}(z, 1, \epsilon), \\
& W^{(2)}\left(z, \xi^{2} / \mu^{2}, \epsilon\right)=\left(\frac{\mu^{2}}{\xi^{2}}\right)^{2 \epsilon} W^{(2)}(z, 1, \epsilon)+\gamma\left(\left(\xi^{2} / \mu^{2}\right)^{\epsilon}, \epsilon\right)\left(\frac{\mu^{2}}{\xi^{2}}\right)^{\epsilon} W^{(1)}(z, 1, \epsilon) .
\end{aligned}
$$

According to eqs. (4.15) and (4.18) the complete one loop result exponentiates in moment space $^{[13]}$, up to possible corrections that behave as $1 / N$. By comparison of eq. (4.15) with the general resummed expression in moment space, eq. (2.20), we observe that we are able to choose $\nu$ in such a way as to absorb all logarithmic $1-z$ dependence into the running coupling. When this is done, leading and next-to-leading logarithms in $N$ will be resummed by the $W^{(1,2)}$ terms in (4.20). We shall make use of this result in the following section. 


\section{Example: Resummation for Inclusive DIS and DY}

As a final topic, we illustrate the simplicity of the resummation method of the previous section, by applying it directly to the Drell-Yan and deeply inelastic scattering cross sections. We go on to verify that the resulting expressions reproduce known finite-order and resummed expressions for the hard-scattering function in the Drell-Yan process in DIS and $\overline{\mathrm{MS}}$ schemes.

Consider first inclusive DIS,

$$
V(q)+h(P) \rightarrow X
$$

where $V$ is a vector boson with spacelike momentum $q$, and $h$ is a hadron. The cross section for this process is customarily expressed in terms of structure functions $F_{i}(i=1,2,3)$. We focus on the case $V=\gamma^{*}$, and the function $F_{2}$. It can be factorized in moment space, as noted in the introduction,

$$
F_{2}^{(h)}\left(N, Q^{2}\right)=\int_{0}^{1} d x x^{N-1} F_{2}\left(x, Q^{2}\right)=\sum_{i} C_{i}\left(N, Q^{2}\right) \phi_{i / h}\left(N, Q^{2}\right),
$$

where $Q^{2}=-q^{2}$ and $x=Q^{2} / 2 P \cdot q$. Here the $C_{i}$ are partonic coefficient functions, and the $\phi_{i / h}$ parton density functions for hadron $h$. (In the following, we denote Mellin transforms by their arguments, and drop the tilde of previous sections.) We will examine $F_{2}^{(h)}$ for large $N$, where the quark contribution $i=q$ is dominant. Henceforth we will denote the contribution of a single quark to $F_{2}^{(h)}$ by $\mathrm{F}_{\mathrm{DIS}}$, dropping the hadron label. By itself, (5.2) does not yet lead to Sudakov exponentiation in moment space. In particular $C$ is still a function of $N$. $\mathrm{F}_{\text {DIS }}$, however, obeys another factorization theorem near the edge of phase space, i.e. at large $N$ (eq. (3.14) in Ref. [13]),

$$
\mathrm{F}_{\text {DIS }}\left(N, Q^{2}\right)=\left|\mathrm{H}_{\text {DIS }}\left(\frac{Q^{2}}{\mu^{2}}\right)\right|^{2} \phi^{\prime}\left(\frac{p_{1} \cdot \zeta_{1}}{\mu}, \frac{Q}{\mu N}\right) J\left(\frac{p_{2} \cdot \zeta_{2}}{\mu}, \frac{Q}{\mu N^{1 / 2}}\right) V\left(\frac{Q}{\mu N}\right)+\mathcal{O}(1 / N),
$$

in terms of a modified quark distribution $\phi^{\prime} . p_{1}$ is the momentum of the incoming hadron (parton, in perturbation theory), and $p_{2}$ the momentum of the scattered quark in the elastic limit. In (5.3), the $N$-dependence of the partonic coefficient function $C_{q}$ and the distribution $\phi_{q / h}$ has been factorized into $\phi^{\prime}$, a hard scattering function $H$, a jet function $J$, corresponding 
to the scattered quark, and a soft function $V$. Each of these functions is infrared safe, but $\phi^{\prime}, J$ and $V$ absorb all of the $N$-dependence, and hence singularities in the elastic limit. The respective $N$ dependences of $\phi^{\prime}, J$ and $V$ may be found from eqs. (4.7), (6.1) and (7.9) of [13]. As above, the $\zeta_{i}$ may be thought of as gauge-fixing vectors used to define the jet functions. The factorized expression (5.3) is of the form of eq. (2.4), and following the arguments of Sections 2 and 4 , this alone is enough to ensure that $F_{\text {DIS }}$ enjoys a Sudakov resummation in logarithms of $N$.

Analogous arguments hold for the Drell-Yan process,

$$
h_{A}\left(p_{1}\right)+h_{B}\left(p_{2}\right) \rightarrow V(q)+X
$$

where $h_{A}$ and $h_{B}$ are hadrons and $V$ is a heavy vector boson $\left(\gamma^{*}, Z, \ldots\right)$ of timelike momentum $q$. The factorization theorem for this process ${ }^{[7]}$ reads in moment space

$$
\begin{aligned}
\int_{0}^{1} d \tau \tau^{N-1} \frac{d \sigma}{d Q^{2}}\left(\tau, Q^{2}\right) & =\sum_{i, j} \phi_{i / A}\left(N, Q^{2}\right) \phi_{j / B}\left(N, Q^{2}\right) \sigma_{i j}\left(N, Q^{2}\right) \\
= & \sum_{q}\left|\mathrm{H}_{\mathrm{DIS}}\left(\frac{Q^{2}}{\mu^{2}}\right)\right|^{2} \psi_{q / A}\left(p_{1} \cdot \zeta_{1}, \frac{Q}{\mu N}\right) U\left(\frac{Q}{\mu N}\right) \psi_{\bar{q} / B}\left(p_{2} \cdot \zeta_{2}, \frac{Q}{\mu N}\right) \\
& +\mathcal{O}(1 / N),
\end{aligned}
$$

where $Q^{2}=q^{2}, \tau \equiv Q^{2} / S$ and $S=\left(p_{1}+p_{2}\right)^{2}$, and the sum is over quarks and antiquarks in the second line. As above, the $\phi_{i / A}\left(x, Q^{2}\right)$ in the first equality are standard parton densities, and $\sigma_{i j}$ is the corresponding partonic DY coefficient function. Furthermore, $\sigma_{i j}\left(N, Q^{2}\right) \equiv$ $\int_{0}^{1} d z z^{N-1} \sigma_{i j}\left(z, Q^{2}\right)$, and similarly for the $\phi$ 's. We restrict ourselves to $i j=q \bar{q}$, which is the dominant production channel at large $N$, and denote the partonic coefficient function for this case by $\sigma_{\mathrm{DY}}$. As for $F_{\mathrm{DIS}}, \sigma_{\mathrm{DY}}(z)$ is singular when $z \rightarrow 1$, but satisfies a further factorization ${ }^{[13]}$, given in the second line of (5.5), in which all of the $\ln N$-dependence of its moment is absorbed into jet functions $\psi$ and a soft function $U$, precisely as in eq. (2.4) above. The explicit $N$-dependeces of $\psi$ and $U$ may be found from eqs. (5.7) and (7.8) of [13].

Finally, the $\overline{\mathrm{MS}}$ distribution ${ }^{[30]}$, which we denote $\phi_{\overline{\mathrm{MS}}}$, may also be treated in a similar fashion. This is because it differs from the $\mathrm{F}_{\mathrm{DIS}}$ as $x \rightarrow 1$ only by replacing the scattered 
quark jet by an outgoing Wilson line ${ }^{[31]}$. We shall observe below, however, certain differences in the resummation of this function, compared to cross sections.

We are now ready to derive specific resummed expressions for the DY and DIS processes, as well as for the $\overline{\mathrm{MS}}$ distribution. We will also determine the exponentiating two-loop coefficients in the hard-scattering function of DY in the DIS and $\overline{\mathrm{MS}}$ factorization schemes.

According to the discussion of Section 4 we need the one loop corrections to $\sigma_{\mathrm{DY}}, \mathrm{F}_{\mathrm{DIS}}$ and $\phi_{\overline{\mathrm{MS}}}$. They are in $d=4-2 \epsilon(\epsilon<0)$ dimensions ${ }^{[32]}$

$$
\begin{aligned}
\sigma_{\mathrm{DY}}^{(1)}\left(z, Q^{2}\right)=- & \frac{\alpha_{s} C_{F}}{\pi}\left(\frac{4 \pi \mu^{2}}{Q^{2}}\right)^{\epsilon} \frac{\Gamma(1-\epsilon)}{\Gamma(1-2 \epsilon)}\left[\delta(1-z)\left(\frac{1}{2}-\frac{\pi^{2}}{3}\right)\right. \\
& \left.+\frac{1}{\epsilon}\left(\frac{1+z^{2}}{(1-z)^{1+2 \epsilon}}\right)_{+} z^{\epsilon}\right], \\
\mathrm{F}_{\mathrm{DIS}}^{(1)}\left(z, Q^{2} / \mu^{2}, \epsilon\right)= & \frac{\alpha_{s} C_{F}}{2 \pi}\left(\frac{4 \pi \mu^{2}}{Q^{2}}\right)^{\epsilon} \frac{\Gamma(1-\epsilon)}{\Gamma(1-2 \epsilon)}\left[\delta(1-z)\left(-\frac{11}{4}-\frac{\pi^{2}}{3}\right)\right. \\
& \left.-\frac{1}{\epsilon}\left(\frac{1+z^{2}+3 \epsilon / 2}{(1-z)^{1+\epsilon}}\right)_{+} z^{\epsilon}+z^{\epsilon}(1-z)^{-\epsilon}(3-z)+3 z\right],
\end{aligned}
$$

and

$$
\phi_{\overline{\mathrm{MS}}}^{(1)}\left(z, Q^{2}\right)=-\frac{\alpha_{s} C_{F}}{2 \pi}\left(\frac{4 \pi \mu^{2}}{Q^{2}}\right)^{\epsilon} \frac{\Gamma(1-\epsilon)}{\Gamma(1-2 \epsilon)} \frac{1}{\epsilon}\left[\frac{1+z^{2}}{1-z}\right]_{+} .
$$

Here $Q^{2}$ denotes the heavy vector boson mass squared in the case of DY, and $-Q^{2}$ the invariant mass of the photon probe in the DIS (and $\overline{\mathrm{MS}}$ ) case; $\mu^{2}$ is the dimensional regularization scale, while $z$ denotes the square of the ratio of the heavy vector boson mass to the parton centerof-mass energy in DY, and the partonic Bjorken scaling variable for DIS and $\overline{\mathrm{MS}}$. The plus distributions in the above expressions are usually expanded as

$$
\left[\frac{1}{(1-z)^{1+\kappa}}\right]_{+}=\left[\frac{1}{(1-z)}\right]_{+}-\kappa\left[\frac{\ln (1-z)}{(1-z)}\right]_{+}+\frac{1}{2} \kappa^{2}\left[\frac{\ln ^{2}(1-z)}{(1-z)}\right]_{+}-\cdots
$$

which we choose not to do here. Redefining $\mu^{2} \rightarrow \mu^{2} \exp \left(-\left(\ln (4 \pi)-\gamma_{E}\right)\right)$ eliminates the $(4 \pi)^{\epsilon}$ in the expressions (5.6), and absorbs the factors $\Gamma(1-\epsilon) / \Gamma(1-2 \epsilon)$. 
Let us exhibit the case of DIS in more detail, as an example. The one loop correction to the DIS partonic structure function leads via eq. (4.18) to $W_{\mathrm{DIS}}^{(1)}(z, 1, \epsilon)$ in momentum space. We can write this kernel as

$$
W_{\mathrm{DIS}}^{(1)}(z, 1, \epsilon)=\delta(1-z) f_{\mathrm{DIS}}^{(1)}(\epsilon)+z^{\epsilon}\left(\frac{g_{\mathrm{DIS}}^{(1)}(z, \epsilon)}{(1-z)^{1+\epsilon}}\right)_{+}+h_{\mathrm{DIS}}^{(1)}(z, \epsilon)
$$

where the coefficient functions $f_{\mathrm{DIS}}^{(1)}, g_{\mathrm{DIS}}^{(1)}, h_{\mathrm{DIS}}^{(1)}$ are regular functions of their arguments at $z=1$, given by

$$
\begin{gathered}
f_{\mathrm{DIS}}^{(1)}(\epsilon)=\frac{C_{F}}{2} \epsilon\left(\frac{11}{4}+\frac{\pi^{2}}{3}\right), \\
g_{\mathrm{DIS}}^{(1)}(z, \epsilon)=\frac{C_{F}}{2}\left(1+z^{2}+\frac{3 \epsilon}{2}+\frac{7 \epsilon^{2}}{2}\right), \\
h_{\mathrm{DIS}}^{(1)}(z, \epsilon)=-\frac{C_{F}}{2} \epsilon\left[z^{\epsilon}(1-z)^{-\epsilon}(3-z)+3 z\right] .
\end{gathered}
$$

We can now use (4.20) to determine the one-loop exponent, and substitute the result into the exponential resummed cross section (4.15).

Given the limitations of the factorization theorem (5.3), we can discard terms of order $1 / N$. Thus we drop $h_{\mathrm{DIS}}^{(1)}(z, \epsilon)$ completely and expand $g_{\mathrm{DIS}}^{(1)}(z, \epsilon)$ around $z=1$, dropping $1 / N$ terms in this expansion where convenient.

According to the discussion below (4.10), the term $\delta(1-z) f_{\mathrm{DIS}}^{(1)}$ and the plus distributions in (5.9) are separately RG invariant. We are therefore free to choose different scales $\nu$ for these two terms in the general resummed expression eq. (4.15). The natural choice for the $\delta(1-z) f_{\mathrm{DIS}}^{(1)}$ term is $\nu=1$ in (4.15). Changes in $\mu$ generate terms $b_{2} \ln \left(\mu^{\prime} / \mu\right)$ at higher orders. The $\lambda$ integral may then be carried out explicitly for this term in (4.15). For the plus distribution term, however, the natural choice is $\nu=1-z$. Then, using $\xi=(1-z)^{1 / 2} \mu$ in eq. (4.20), we absorb the factor $(1-z)^{-\epsilon}$ in (5.9) into the limit of the $\lambda$ integral in eq. (4.15), involving only the running coupling, as indicated at the end of Sec. 4. 
Treating the $N$-independent term $f^{(1)}$ in this fashion, we obtain for the one-loop resummation of the DIS cross section,

$$
\begin{aligned}
\mathrm{F}_{\text {DIS }}\left(N, Q^{2} / \mu^{2}, \alpha\left(\mu^{2}\right), \epsilon\right) \\
=\exp \left[\frac{C_{F}}{2} \int_{0}^{1} d z \frac{z^{N-1+\epsilon}-1}{1-z}\left\{\int_{0}^{Q^{2}(1-z) / \mu^{2}} \frac{d \lambda}{\lambda}\left[\left(1+z^{2}\right)+\frac{3 \epsilon}{2}\right] \alpha\left(\lambda, \alpha\left(\mu^{2}\right), \epsilon\right)\right\}\right] \\
\times \exp \left[-\frac{\alpha\left(\mu^{2}\right) C_{F}}{2}\left(11 / 4+\pi^{2} / 3\right)\right] \\
=\exp \left[\frac { C _ { F } } { 2 } \int _ { 0 } ^ { 1 } d z \frac { z ^ { N - 1 + \epsilon } - 1 } { 1 - z } ( 1 + z ^ { 2 } ) \left\{\int_{0}^{Q^{2}(1-z) / \mu^{2}} \frac{d \lambda}{\lambda} \alpha\left(\lambda, \alpha\left(\mu^{2}\right), \epsilon\right)\right.\right. \\
\left.\quad-\frac{3}{2} \alpha\left(Q^{2}(1-z) / \mu^{2}, \alpha_{s}\left(\mu^{2}\right), \epsilon\right)\right\} \\
\quad \times \exp \left[-\frac{\alpha\left(\mu^{2}\right) C_{F}}{2}\left(11 / 4+\pi^{2} / 3\right)\right]
\end{aligned}
$$

where in the second form we have evaluated the $3 \epsilon / 2$ term to lowest order in $\alpha_{s}\left(Q^{2}(1-z)\right)$. Note that as long as were are in $d$ dimensions the $\lambda$ integrals in the exponent are well-defined at their lower limits. There is no particular problem with integrals dominated by their lower limits here, since $F_{\text {DIS }}$ is not by itself infrared safe.

For the Drell-Yan cross section one obtains by essentially identical methods,

$$
\begin{aligned}
\sigma_{\mathrm{DY}} & \left(N, Q^{2} / \mu^{2}, \alpha\left(\mu^{2}\right), \epsilon\right) \\
= & \exp \left[C_{F} \int_{0}^{1} d z\left(\frac{z^{N-1+\epsilon}-1}{1-z}\right)\left(1+z^{2}\right)\right. \\
& \times \exp \left[-\alpha\left(\mu^{2}\right) C_{F}\left(1 / 2-\pi^{2} / 3\right)\right] .
\end{aligned}
$$

Here, the natural choice of scale for the plus distributions is $\nu=(1-z)^{2}$.

It is not possible to treat the $\overline{\mathrm{MS}}$ quark distribution $\phi_{\overline{\mathrm{MS}}}$ in quite the same way, because it is not normalized at $Q^{2}=0$ by electroweak current conservation. Indeed, it is usually regarded as a function of only a single scale, through the running coupling. Nevertheless, it satisfies an evolution equation ${ }^{[26]}$ of precisely the form (4.3), with $W_{\overline{\mathrm{MS}}}$ given by the standard 
splitting functions. ${ }^{[31]}$ The problem we must therefore solve is how to fix a boundary condition for this equation. To this end, we note that for $\epsilon<0(d>4)$, the dimensionally-continued running coupling vanishes at zero scale, $\alpha\left(0, \alpha\left(\mu_{1}^{2}\right), \epsilon\right)=0$, order by order in its perturbative expansion in the coupling $\alpha\left(\mu_{1}^{2}\right)$ evaluated at any nonzero scale $\mu_{1}$. Dimensionally-continued radiative corrections to $\phi_{\overline{\mathrm{MS}}}\left(N, \alpha\left(Q^{2}\right)\right)$ therefore vanish at $Q^{2}=0$, and we may take, just as for the electroweak cross sections above, $\phi_{\overline{\mathrm{MS}}}\left(N, Q^{2}=0, \epsilon\right)=0$. The solution to the evolution equation is then fixed by this boundary condition, and we find

$$
\begin{aligned}
\phi_{\overline{\mathrm{MS}}} & \left(N, Q^{2}, \epsilon\right) \\
& =\exp \left[-\int_{0}^{Q^{2}} \frac{d \mu^{\prime 2}}{\mu^{\prime 2}} \Gamma_{q q}\left(N, \alpha_{s}\left(\mu^{\prime 2}\right)\right)\right] \\
& =\exp \left[\frac{C_{F}}{2} \int_{0}^{1} d z\left(\frac{z^{N-1}-1}{1-z}\right)\left(1+z^{2}\right) \int_{0}^{Q^{2} / \mu^{2}} \frac{d \lambda}{\lambda} \alpha\left(\lambda, \alpha\left(\mu^{2}\right), \epsilon\right)+\ldots\right] .
\end{aligned}
$$

In the first equality, $\Gamma_{q q}(N, \alpha)$ are the (singular, diagonal) terms in the quark anomalous dimension matrix, which contribute $\ln N$ dependence. In the second equality, we have introduced an arbitrary scale $\mu^{2}$ through $\lambda \equiv \mu^{\prime 2} / \mu^{2}$, for which $\alpha\left(\lambda, \alpha\left(\mu^{2}\right), \epsilon\right)=\alpha\left(\mu^{\prime 2}\right)$. This brings our expression into the same form as eqs. (5.13) and (5.14).

The expressions for $\mathrm{F}_{\mathrm{DIS}}, \phi_{\overline{\mathrm{MS}}}$ and $\sigma_{\mathrm{DY}}$ in eqs. (5.13), (5.15) and (5.14) are singular as $\epsilon \rightarrow 0$. However, this singular behavior is universal for all three quantities. Thus we can perform mass factorization to obtain the finite Drell-Yan hard part, which is a simple ratio in moment space:

$$
\omega_{q \bar{q}}\left(N, Q^{2} / \mu^{2}, \alpha\left(\mu^{2}\right)\right)=\frac{\sigma_{\mathrm{DY}}\left(N, Q^{2} / \mu^{2}, \alpha\left(\mu^{2}\right), \epsilon\right)}{\phi^{2}\left(N, Q^{2} / \mu^{2}, \alpha\left(\mu^{2}\right), \epsilon\right)}
$$

where $\phi(N)$ is the moment of a suitable quark distribution (mixing with other partons is down by $1 / N)$. Choosing for $\phi$ alternatively $\mathrm{F}_{\mathrm{DIS}}$ and $\phi_{\overline{\mathrm{MS}}}$ in (5.16) yields the finite resummed hard part for the Drell-Yan cross section in the DIS and $\overline{\mathrm{MS}}$ schemes. The factorization scale is set by the denominator, and we choose it to be $Q^{2}$. Putting $\mu^{2}=Q^{2}$, and returning to four 
dimensions because $\omega$ is infrared safe, we have finally

$$
\begin{aligned}
\omega_{q \bar{q}}^{\mathrm{DIS}}\left(N, \alpha\left(Q^{2}\right)\right)= & \exp \left[\int _ { 0 } ^ { 1 } d z ( \frac { z ^ { N - 1 } - 1 } { 1 - z } ) \left\{\left(1+z^{2}\right) \int_{(1-z)^{2}}^{(1-z)} \frac{d \lambda}{\lambda}\left(-2 C_{F}\right) \alpha\left(\lambda Q^{2}\right)\right.\right. \\
& \left.\left.+\frac{3 C_{F}}{2} \alpha\left((1-z) Q^{2}\right)\right\}\right] \\
& \times \exp \left[\frac{\alpha\left(Q^{2}\right) C_{F}}{2}\left(9 / 2+4 \pi^{2} / 3\right)\right],
\end{aligned}
$$

and

$$
\begin{aligned}
\omega_{q \bar{q}}^{\overline{\mathrm{MS}}}\left(N, \alpha\left(Q^{2}\right)\right) & =\exp \left[\int_{0}^{1} d z\left(\frac{z^{N-1}-1}{1-z}\right)\left(1+z^{2}\right) \int_{(1-z)^{2}}^{1} \frac{d \lambda}{\lambda}\left(-2 C_{F}\right) \alpha\left(\lambda Q^{2}\right)\right] \\
& \times \exp \left[\frac{-\alpha\left(Q^{2}\right) C_{F}}{2}\left(1-2 \pi^{2} / 3\right)\right] .
\end{aligned}
$$

One may check that expansion to one loop reproduces the one loop hard parts in [32], up to terms of order $1 / N$. We denote the $N$-independent terms in the above expressions by $A\left(\alpha\left(Q^{2}\right)\right) .^{\star}$ Then both of the above results are of the form

$$
\omega_{q \bar{q}}^{S}\left(N, \alpha\left(Q^{2}\right)\right)=A_{S}\left(\alpha\left(Q^{2}\right)\right) I_{S}\left(N, \alpha\left(Q^{2}\right)\right),
$$

where the $N$-dependent exponential $I_{S}$ is

$$
\begin{aligned}
& I_{S}\left(N, \alpha\left(Q^{2}\right)\right)= \\
& \exp \left[-\int_{0}^{1} d z \frac{z^{N-1}-1}{1-z}\left\{\int_{(1-z)^{2}}^{(1-z)^{m_{S}}} \frac{d \lambda}{\lambda} g_{1}\left[\alpha\left(\lambda Q^{2}\right)\right]+g_{2}\left[\alpha\left((1-z)^{m_{S}} Q^{2}\right)\right]\right\}\right] .
\end{aligned}
$$

Here $m_{S}=1,0$ for the DIS and $\overline{\mathrm{MS}}$ schemes respectively, and the functions $g_{1}, g_{2}$ have finite expansions in their arguments:

$$
g_{1}[\alpha]=\sum_{n=1}^{\infty} \alpha^{n} g_{1}^{(n)}, g_{2}[\alpha]=\sum_{n=1}^{\infty} \alpha^{n} g_{2}^{(n)} .
$$

The expression (5.20) organizes all large logarithms $(\ln N$ or $\ln (1-z))$ in the perturbation expansion for $\omega_{q \bar{q}}^{S}$. Its expansion in $\alpha\left(Q^{2}\right)$ generates terms $\alpha^{n}\left(Q^{2}\right) \ln ^{m} N, n=0, . ., \infty, m \leq$

$\star$ Note that they may be numerically quite important. 
$n+1$ in the exponent. To be specific, for a given order $n$, one can call terms for which $m=n+1$ "leading logarithmic", terms with $m=n$ "next-to-leading", and ones where $m<n$ "subdominant". In this terminology $g_{1}^{(1)}$ contributes at the leading logarithmic level, $g_{2}^{(1)}$ and $g_{1}^{(2)}$ at the next-to-leading level, while $g_{2}^{(2)}$ is subdominant.

The form (5.20) was derived via different methods in refs. [13] and [14] for the DIS scheme, and for the $\overline{\mathrm{MS}}$ scheme in Ref. [33]. The lowest order coefficients are obvious from (5.17) and (5.18):

$$
\begin{gathered}
\left.g_{1}^{(1)}\right|_{\mathrm{DIS}}=2 C_{F},\left.g_{2}^{(1)}\right|_{\mathrm{DIS}}=-3 C_{F} / 2 \\
\left.g_{1}^{(1)}\right|_{\overline{\mathrm{MS}}}=2 C_{F},\left.g_{2}^{(1)}\right|_{\overline{\mathrm{MS}}}=0,
\end{gathered}
$$

and agree with those found in [13], [14] and [33]. Although, strictly speaking, we have not proved here that the form (5.19) holds for all $g_{i}^{(j)}$, functions of different arguments for the running coupling can always be brought to the above form, by the method discussed in Ref. [15]. In particular, the argument of $\alpha_{s}$ in $g_{2}$ in eq. (5.20) is arbitrary in the $\overline{\mathrm{MS}}$ scheme, up to subdominant terms, because $\left.g_{2}^{(1)}\right|_{\overline{\mathrm{MS}}}=0$.

We may improve the accuracy of our resummation by determining the coefficients $g_{i}^{(2)}$ in both schemes. We do this by expanding (5.20) to order $\alpha^{2}$ and comparing with the explicit two-loop results in [34], which is most conveniently done in moment space. Using methods described in [35], we find, as in [14], [35],

$$
\left.g_{1}^{(2)}\right|_{\mathrm{DIS}}=\left.g_{1}^{(2)}\right|_{\overline{\mathrm{MS}}}=\gamma_{K}^{(2)}=C_{A} C_{F}\left(\frac{67}{18}-\zeta(2)\right)+n_{f} C_{F}\left(-\frac{5}{9}\right)
$$

the two loop anomalous dimension first identified by Kodaira and Trentadue ${ }^{[36][37][5][20]}$, and

$$
\begin{aligned}
&\left.g_{2}^{(2)}\right|_{\mathrm{DIS}}=C_{F}^{2}\left(-\frac{3}{16}+\frac{3}{2} \zeta(2)-3 \zeta(3)\right)+C_{A} C_{F}\left(-\frac{57}{16}-\frac{11}{6} \zeta(2)+\frac{3}{2} \zeta(3)\right) \\
&+ n_{f} C_{F}\left(\frac{5}{8}+\frac{1}{3} \zeta(2)\right) \\
&\left.g_{2}^{(2)}\right|_{\mathrm{MS}}=C_{A} C_{F}\left(\frac{101}{27}-\frac{11}{3} \zeta(2)-\frac{7}{2} \zeta(3)\right)+n_{f} C_{F}\left(-\frac{14}{27}+\frac{2}{3} \zeta(2)\right) .
\end{aligned}
$$

The numerical impact of the latter coefficients is quite small near threshold, as they contribute only at the sub-dominant logarithmic level. 


\section{Conclusion}

We have presented a unified method for resumming Sudakov corrections to partonic cross sections. We assumed the existence of a factorized expression (2.4), which is applicable to a large class of cross sections in the "elastic limit" of phase space, where their QCD corrections due to soft gluons can be large. To factorize the cross section in these regions, it is in general necessary to introduce dependence on auxiliary vectors $\zeta_{i}$. The freedom in choosing the $\zeta_{i}$ leads to consistency equations which resum the large corrections.

To illustrate the method we have rederived the large $x$ resummed Drell-Yan hard parts in DIS and $\overline{\mathrm{MS}}$ factorization schemes. These expressions are relevant to both perturbative resummation and power corrections. For example, in [16], the $z$ integral in (5.19) was treated by a principal value presciption ${ }^{[38]}$ for the Drell-Yan process in DIS scheme, to define the inte-

gral in the presence of its renormalon pole ${ }^{[21]}$. It was observed that in resummed perturbation theory the pole manifests itself only at the level of power-suppressed ("higher twist") effects. The precise nature of this dependence is an active subject of inquiry. Comparisons with data using the resummed (differential) DY cross section have also been carried out. ${ }^{[17]}$ Other questions of theoretical interest may include the application of effective field theory methods to Sudakov factorization. Phenomenologically interesting applications are possible to any QCD reaction for which soft gluon resummation is relevant, such as high $p_{t}$ jet production and high mass heavy quark production. ${ }^{[24[22]}$ We hope that the streamlined discussion of Sudakov resummation given above will facilitate future inquiry in this area.

Acknowledgements. We would like to thank Lyndon Alvero, Ed Berger, John Collins, Nicholaos Kidonakis, Gregory Korchemsky, Al Mueller, Jack Smith, Dave Soper and WuKi Tung for helpful conversations. This work was supported in part by the National Science Foundation under grant PHY9309888, and by the U.S. Department of Energy under contract W-31-109-ENG-38. 


\section{REFERENCES}

1. A.H. Mueller, Phys. Rev. D20 (1979) 2037.

2. J.C. Collins and D.E. Soper, Nucl. Phys. B193 (1981) 381.

3. A. Sen, Phys. Rev. D24 (1981) 3281.

4. V.V Sudakov, Sov. Phys. JETP 3 (1956) 65; M. Cassandro and M. Cini, Nuovo Cimento 34 (1964) 1719; R. Jackiw, Ann. Phys. (N.Y.) 48 (1968) 292; P.M. Fishbane and J.D. Sullivan, Phys. Rev. D4 (1971) 458.

5. J.C. Collins, in Perturbative Quantum Chromodynamics, ed. A.H. Mueller (World Scientific, Singapore, 1989).

6. L. Magnea and G. Sterman, Phys. Rev. D42 (1990) 4222.

7. J.C. Collins, D.E. Soper and G. Sterman, in Perturbative Quantum Chromodynamics, ed. A.H. Mueller (World Scientific, Singapore, 1989).

8. J.C. Collins, D.E. Soper and G. Sterman, Nucl. Phys. B261 (1985) 104; Nucl. Phys. B308 (1988) 833.

9. G.T. Bodwin, Phys. Rev. D31 (1985) 2616.

10. K.G. Wilson and J. Kogut, Phys. Rep. 12 (1974) 75; J. Polchinski, Nucl. Phys. B231 (1984) 269; H. Georgi, Ann. Rev. Nucl. Part. Sci., 43 (1993) 209.

11. J.C. Collins and D.E. Soper, Nucl. Phys. B197 (1982) 446.

12. J.C. Collins, D.E. Soper and G. Sterman, Nucl. Phys. B250 (1985) 199; G. Altarelli, R.K. Ellis, M. Greco and G. Martinelli, Nucl. Phys. B246 (1984) 12; C.T.H. Davies and W.J. Stirling, Nucl. Phys. B244 (1984) 337; C.T.H. Davies, B.R. Webber and W.J. Stirling, Nucl. Phys. B256 (1985) 413; P.B. Arnold and R.P. Kauffman, Nucl. Phys. B349 (1991) 381; G.A. Ladinsky and C.P. Yuan, Phys. Rev. 50 (1994) R4239.

13. G. Sterman, Nucl. Phys. B281 (1987) 310.

14. S. Catani and L. Trentadue, Nucl. Phys. B327 (1989) 323.

15. S. Catani and L. Trentadue, Nucl. Phys. B353 (1991) 183. 
16. H. Contopanagos and G. Sterman, Nucl. Phys. B400 (1993) 211; 419 (1994) 77; L. Alvero and H. Contopanagos, Nucl. Phys. B436 (1995) 184.

17. L. Alvero and H. Contopanagos, Nucl. Phys. B456 (1995) 497.

18. S. Catani, L. Trentadue, G. Turnock and B.R. Webber, Nucl. Phys. B407 (1993) 3.

19. J. Botts and G. Sterman, Nucl. Phys. B325 (1989) 62.

20. G.P. Korchemsky and A.V. Radyushkin, Phys. Lett. B171 (1986) 459; I.A. Korchemskaya and G.P. Korchemsky, Nucl. Phys. B437 (1995) 127; J.G.M. Gatheral, Phys. Lett. B113 (1983) 90; J. Frenkel and J.C. Taylor, Nucl. Phys. B246 (1984) 231.

21. D. Appell, P. Mackenzie and G. Sterman in Proceedings of the Storrs Meeting, Fourth Meeting of the Division of Particles and Fields, Storrs, CT, August 15-18, 1988, p. 567; B.R. Webber, Phys. Lett. B339 (1994) 148; G.P. Korchemsky and G. Sterman, Phys. Lett. B340 (1994) 96; Nucl. Phys. B437 (1995) 415; A.V. Manohar and M.B. Wise, Phys. Lett. B344 (1995) 407; Yu.L. Dokshitzer and B.R. Webber, Phys. Lett. B352 (1995) 451; R. Akhoury and V.I. Zakharov, Phys. Lett. B357 (1995) 646; P. Nason and M.H. Seymour, Nucl. Phys. B454 (1995) 291; M. Beneke and V.M. Braun, Nucl. Phys. B454 (1995) 253.

22. N. Kidonakis and G. Sterman, Stony Brook preprint ITP-SB-96-7 (April, 1996), hep$\mathrm{ph} / 9604234$.

23. G. Tiktopoulos, Nucl. Phys. B147 (1979) 371; G. Sterman, Phys. Rev. D19 (1979), 3135.

24. E. Laenen, J. Smith and W.L. van Neerven, Nucl. Phys. B369 (1992) 543; Phys. Lett. B321 (1994) 254; E.L. Berger and H. Contopanagos, Phys. Lett. B361 (1995) 115; Argonne preprint ANL-HEP-95-82, hep-ph/9603326; N. Kidonakis and J. Smith, Phys. Rev. D51 (1995) 6092; S. Catani, M.L. Mangano, P. Nason and L. Trentadue, CERN preprint CERN-TH/96-21, hep-ph/9602208.

25. M.G. Sotiropoulos and G. Sterman, Nucl Phys. B419 (1994) 59; B425 (1994) 489; G.P. Korchemsky, Phys. Lett. B325 (1994) 459; I.A. Korchemskaya and G.P. Korchemsky, Ref. [20]. 
26. G. Altarelli and G. Parisi, Nucl. Phys. B126 (1977) 298; V.N. Gribov and L.N. Lipatov, Sov. J. Nucl. Phys. 15 (1972) 438, 675; Yu.L. Dokshitser, Sov. Phys. JETP 46 (1977) 641.

27. H. Contopanagos and G. Sterman, Ref. [16].

28. G. Altarelli, R.K. Ellis, M. Greco and G. Martinelli, Ref. [12].

29. G.P. Korchemsky and G. Sterman, Ref. [21].

30. W.A. Bardeen, A.J. Buras, D.W. Duke and T. Muta, Phys. Rev. D18 (1978) 3998.

31. J.C. Collins and D.E. Soper, Nucl. Phys. B194 (1982) 445.

32. G. Altarelli, R.K. Ellis and G. Martinelli, Nucl. Phys. B143 (1978) 521, (E)146 (1978) 544, B157 (1979) 461.

33. S. Catani, G. Marchesini, B.R. Webber, Nucl. Phys. B349 (1991) 635.

34. T. Matsuura, S.C. van der Marck and W.L. van Neerven, Nucl. Phys. B319 (1989) 570;

R. Hamberg, W.L. van Neerven and T. Matsuura, Nucl. Phys. B359 (1991) 343.

35. L. Magnea, Nucl. Phys. B349 (1991) 703.

36. J. Kodaira and L. Trentadue, Phys. Lett. B112 (1982) 66.

37. C.T.H. Davies and W.J. Stirling, in Ref. [12].

38. A.H. Mueller, Nucl. Phys. B250 (1985) 327. 


\section{FIGURE CAPTION}

Figure 1. (a) Cut diagram representing the factorization of eq. (2.4) in $\mathrm{e}^{+} \mathrm{e}^{-}$annihilation in the elastic limit; (b) same for DIS; (c) same for Drell-Yan. 


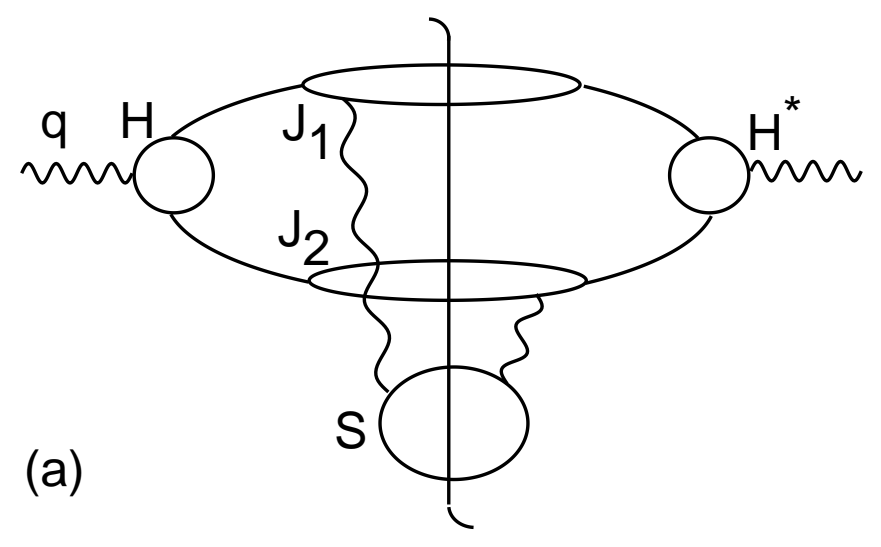

(b)
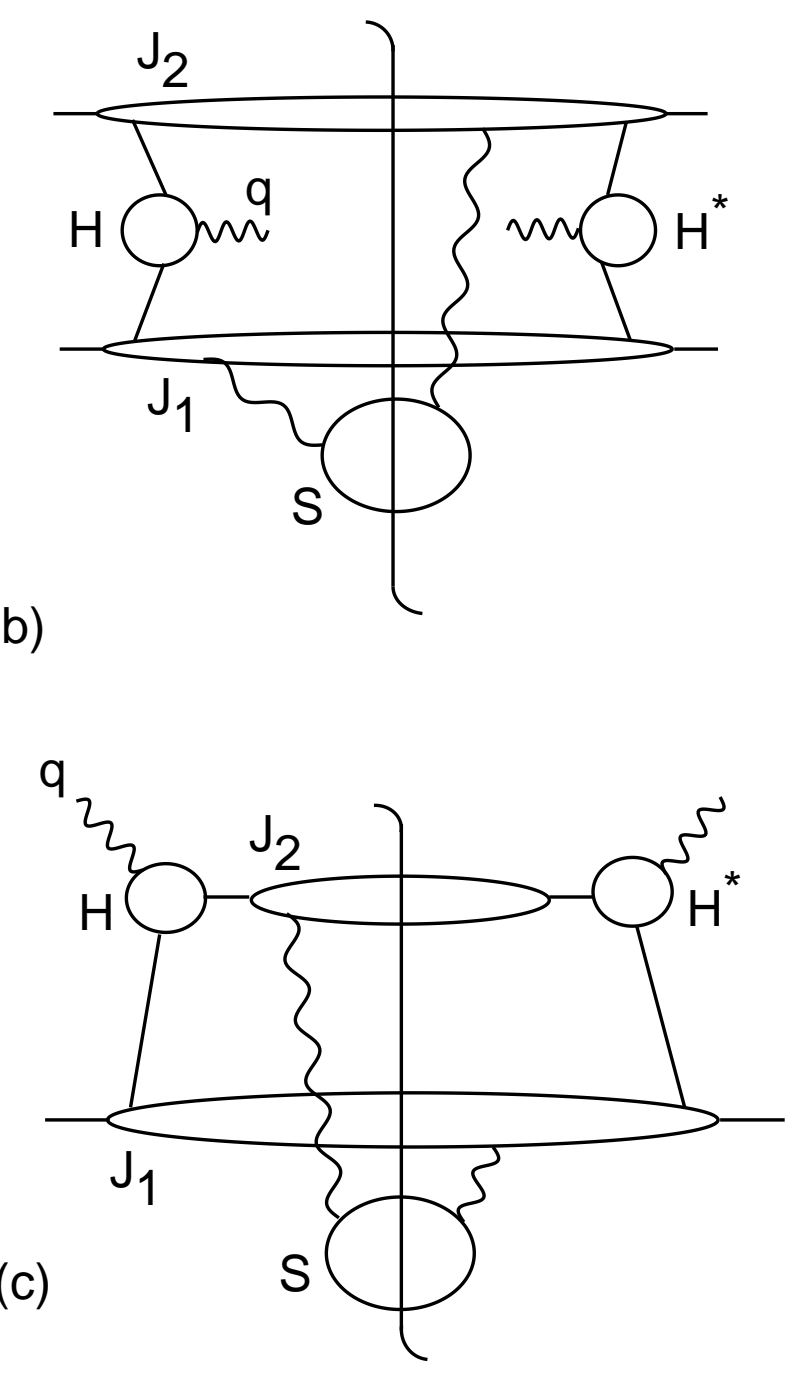